\title{
Simultaneous copy number gains of NUPR1 and ERBB2 predicting poor prognosis in early-stage breast cancer
}

Seung-Hyun Jung ${ }^{1,2}$, Ahwon Lee ${ }^{3}$, Seon-Hee Yim ${ }^{1,4}$, Hae-Jin Hu ${ }^{1,2}$, Chungyoul Choe ${ }^{1,2}$ and Yeun-Jun Chung ${ }^{1,2^{*}}$

\begin{abstract}
Background: The full extent of chromosomal alterations and their biological implications in early breast carcinogenesis has not been well examined. In this study, we aimed to identify chromosomal alterations associated with poor prognosis in early-stage breast cancers (EBC).

Methods: A total of 145 EBCs (stage I and II) were examined in this study. We analyzed copy number alterations in a discovery set of 48 EBCs using oligoarray-comparative genomic hybridization. In addition, the recurrently altered regions (RARs) associated with poor prognosis were validated using an independent set of 97 EBCs.

Results: A total of 23 RARs were defined in the discovery set. Six were commonly detected in both stage I and II groups (> 50\%), suggesting their connection with early breast tumorigenesis. There were gains on 1q21.2-q21.3, $8 q 24.13,8 q 24.13-21,8 q 24.3$, and 8q24.3 and a loss on 8p23.1-p22. Among the 23 RARs, copy number gains on 16p11.2 (NUPR1) and 17q12 (ERBB2) showed a significant association with poor survival $(P=0.0186$ and $P=0.0186$, respectively). The patients simultaneously positive for both gains had a significantly worse prognosis $(P=0.0001)$. In the independent replication, the patients who were double-positive for NUPR1-ERBB2 gains also had a significantly poorer prognosis on multivariate analysis ( $H R=7.31,95 \% \mathrm{Cl} 2.65-20.15, P=0.0001)$.

Conclusions: The simultaneous gain of NUPR1 and ERBB2 can be a significant predictor of poor prognosis in EBC. Our study will help to elucidate the molecular mechanisms underlying early-stage breast cancer tumorigenesis. This study also highlights the potential for using combinations of copy number alterations as prognosis predictors for EBC.
\end{abstract}

\section{Background}

Breast cancer is the most common female cancer and the leading cause of cancer-related mortality in women worldwide [1,2]. Due to mammographic screening and advances in chemotherapy, breast cancer mortality rates have decreased in developed countries since 1990 [3,4]. Nonetheless, axillary-node negative patients treated by surgery showed a ten-year recurrence rate of approximately $20 \%$ [5]. The five-year survival rate of stage I and II breast cancer patients is reported to be approximately $80 \%$ to $88 \%$ [6-8]. This means that $10-20 \%$ of early-stage

\footnotetext{
* Correspondence: yejun@catholic.ac.kr

'Integrated Research Center for Genome Polymorphism, College of Medicine, The Catholic University of Korea, 505 Banpo-dong, Socho-gu, Seoul 137-701, Republic of Korea

${ }^{2}$ Department of Microbiology, The Catholic University of Korea, Seoul, South Korea

Full list of author information is available at the end of the article
}

breast cancer $(\mathrm{EBC})$ patients have poor clinical outcomes. When considering the large impact that breast cancer has on public health, it is worth investigating genetic mechanisms underlying poor clinical outcomes of some EBCs.

Genomic instability is one of the hallmarks of breast cancer. DNA copy number aberrations, commonly detected phenomena in cancer lesions, are thought to be involved in tumorigenesis and to affect cancer phenotypes [9]. Different patterns of copy number alterations are associated with distinct gene expression patterns and clinical characteristics of breast cancer [10]. A number of chromosomal alterations and subsequent expression changes have been investigated to determine their implications in clinical phenotypes or prognosis. These investigations have resulted in the identification of some cancer-related genes in breast cancer [11]. For example, 
HER2 amplification/overexpression is known to occur at an early developmental stage of ductal carcinoma in situ (DCIS). Loss of 16q, where potential tumor suppressor genes such as E-cadherin $(C D H 1)$ and $C D H 13$ are located, is also known to be a major event in low-grade invasive ductal carcinoma [12,13]. Especially, recent larger-scale studies have elucidated the molecular complexity of breast cancer and suggested novel genetic subgroups [14-18]. However, since most of them studied Caucasians or Hispanics, the profiles of chromosomal alterations and their biological implications in Asians are relatively less well studied.

In this study, we aimed to describe commonly occurring chromosomal alterations in EBC (stage I and II) and to explore the implications of recurrently altered regions (RAR) on patient prognosis. For this purpose, we analyzed DNA copy number alterations (CNAs) across the whole genome using oligoarray-comparative genomic hybridization $(\mathrm{CGH})$ in a discovery set of EBC patients. RARs in the discovery set that were found to be significantly associated with prognosis were validated in an independent replication set. Our results will contribute to a better understanding of early tumorigenesis in breast cancer and will help to predict the prognosis of EBC patients.

\section{Methods}

\section{Patients and tumor specimens}

As a discovery set for the whole genome array-CGH analysis, frozen tumor tissues were obtained from 48 EBC patients who underwent surgical resection at Dankook University Hospital in Cheonan, Korea (from 1998 to 2002). As an independent replication set, 97 formalinfixed, paraffin-embedded (FFPE) EBC tissue samples (from 1996 to 2002) were obtained from Seoul St. Mary's Hospital, Korea. Patient survival status was obtained in 2010 from the Korean Central Cancer Registry, Ministry of Health and Welfare, Korea. All breast cancers were stage I, IIA, or IIB. This study was performed under approval from the Institutional Review Board of the Catholic University Medical College of Korea (CUMC06U015). Tumor stage was determined according to the standard AJCC guidelines for tumor-node-metastasis classification (sixth edition). Clinicopathologic characteristics of the study subjects are summarized separately for the discovery and replication sets in Table 1 . Hormone receptor status for ER, PR and HER2 was obtained through a medical record review and for the cases without the hormone receptor status, immunohistochemical (IHC) staining for ER, PR and HER2 was performed. Based on the IHC measurement, breast cancer cases were categorized into four different molecular subtypes as described elsewhere: luminal type A (ER + and/or PR +, HER2 -: Luminal A), luminal type $\mathrm{B}(\mathrm{ER}+$ and/or $\mathrm{PR}+$, HER2 +: Luminal B), Her2
Table 1 General characteristics of the study subjects

\begin{tabular}{|c|c|c|}
\hline Characteristics & $\frac{\text { Discovery set }}{(n=48)}$ & $\frac{\text { Replication set }}{(n=97)}$ \\
\hline \multicolumn{3}{|l|}{ Age group } \\
\hline$<50$ years & $26(54.2 \%)$ & $22(45.8 \%)$ \\
\hline$\geq 50$ years & $48(49.5 \%)$ & $49(50.5 \%)$ \\
\hline \multicolumn{3}{|l|}{ Stage } \\
\hline Stage I & $11(22.9 \%)$ & $25(25.8 \%)$ \\
\hline Stage II & $37(77.1 \%)$ & $72(74.2 \%)$ \\
\hline Stage IIA & 26 & 50 \\
\hline Stage IIB & 11 & 22 \\
\hline \multicolumn{3}{|l|}{ ER status } \\
\hline Positive & 25(52.1\%) & $54(55.7 \%)$ \\
\hline Negative & 23(47.9\%) & $43(44.3 \%)$ \\
\hline \multicolumn{3}{|l|}{ PR status } \\
\hline Positive & $35(72.9 \%)$ & $59(60.8 \%)$ \\
\hline Negative & 13(27.1\%) & $38(39.2 \%)$ \\
\hline \multicolumn{3}{|l|}{ HER2 status } \\
\hline Positive & $11(22.9 \%)$ & $23(23.7 \%)$ \\
\hline Negative & $37(77.1 \%)$ & $74(76.3 \%)$ \\
\hline \multicolumn{3}{|l|}{ Subtype } \\
\hline Luminal A & $29(60.4 \%)$ & $53(54.6 \%)$ \\
\hline Luminal B & 10(20.8\%) & 16(16.5\%) \\
\hline HER2 & $1(2.1 \%)$ & $7(7.2 \%)$ \\
\hline TNBC & $8(16.7 \%)$ & $21(21.6 \%)$ \\
\hline
\end{tabular}

overexpressed (ER - and PR -, HER2 +: HER2), and triple negative (ER -/PR -/HER2 -: TNBC) [19]. For array-CGH analysis, $10-\mu$ m-thick frozen sections of tumor cell-rich areas (>60\%) were microdissected. Genomic DNA was extracted from these sections using a DNeasy Blood \& Tissue Kit (Qiagen, Hilden, Germany). For genomic real-time quantitative PCR (qPCR) analysis, $10-\mu \mathrm{m}$-thick paraffin sections of tumor cell-rich areas $(>60 \%)$ in the replication set were microdissected. After paraffin removal, genomic DNA was extracted using a DNeasy Blood \& Tissue Kit (Qiagen). Genomic DNA from a healthy female individual was used as the normal reference for all array-CGH experiments. Genomic DNA extracted from the blood of a Korean female individual without breast cancer was used as universal normal reference for all the array-CGH experiments.

\section{Array-CGH and data processing}

For array-CGH analysis, 30K whole-genome human oligoarrays (Human OneArray ${ }^{\mathrm{TM}}$; Phalanx Biotech, Palo Alto, CA) were used. Oligoarray-CGH was performed as described elsewhere [20]. In brief, $1 \mu \mathrm{g}$ of genomic DNA from tumor tissue was labeled with Cy3-dCTP. The reference DNA was labeled with Cy5-dCTP (GeneChem, 
Daejon, Korea). Dye-labeled DNA was purified with BioPrime spin columns (Invitrogen, Carlsbad, CA) and precipitated with $100 \mu \mathrm{g}$ of human Cot-1 DNA (ConnectaGen, Seoul, Korea). The labeled DNA pellet was dissolved in $50 \mu \mathrm{l}$ of DIG hybridization buffer (Roche, Mannheim, Germany), to which $600 \mu \mathrm{g}$ of yeast t-RNA (Invitrogen) was added. The labeled DNA solution was applied on the array and incubated for 48 hours at $37^{\circ} \mathrm{C}$ in a MAUI hybridization machine (BioMicro, Salt Lake City, UT). After washing the slides, arrays were scanned using a GenePix 4000B scanner (Axon Instruments, Sunnyvale, CA) and feature extraction was performed using GenePix Pro 6.0. Normalization and realignment of raw array $\mathrm{CGH}$ data were performed using the web-based array CGH analysis interface, ArrayCyGHt [21]. A print-tip Loess normalization method was used and each probe was mapped according to its genomic location in the UCSC genome browser (Human NCBI36/hg18). In total, 24,107 probes were processed out of initial 26,616 probes. Array-CGH data for all 48 cancers are available through GEO (accession no GSE37839).

\section{Detection of recurrent copy number alterations}

The rank-segmentation statistical algorithm in NEXUS software v3.1 (BioDiscovery Inc., El Segundo, CA) was used to define CNAs of each sample. To optimize the algorithmic parameters for calling CNAs, 11 independent normal-to-normal hybridizations were performed (10 self-to-self and 1 male-to-female hybridizations). The parameters for defining CNAs were as follows: significance threshold $=5.0 \mathrm{E}-4$; maximum contiguous probe spacing $(\mathrm{Kbp})=1000$; minimum number of contiguous probes per CNA segment $=5$; threshold of signal intensity ratio $>0.2$ on $\log _{2}$ scale for gains and $<-0.3$ on $\log _{2}$ scale for losses. After defining CNAs, RAR was determined to be the chromosomal segment covering overlapping CNAs that appeared in at least 30\% of the samples with $P<0.05$ in the discovery set (NEXUS software v3.1). High-level amplification (amplification hereafter) was defined as a probe signal intensity ratio of 1.5 or higher on the $\log _{2}$ scale. Likewise, a homozygous deletion (HD) was defined as a ratio of -1.5 or lower on the $\log _{2}$ scale.

\section{Genomic quantitative PCR analysis}

qPCR validation of the significant RARs was performed using genomic DNA extracted from the FFPE samples of 97 EBCs. As a diploid internal control, a genomic region on chromosome13 (13q32.1) that showed no genomic alteration in the array-CGH data was used. Details including primer information for targets and the diploid control locus are available in Additional file 1. Genomic qPCR was performed using the Mx3000P qPCR system
(Stratagene, La Jolla, CA), as described elsewhere [22]. In brief, a 10- $\mu$ l real-time qPCR mixture containing 10 ng of genomic DNA, SYBR Premix Ex Taq II ${ }^{\mathrm{TM}}$ (TaKaRa Bio, Japan), $1 \times$ ROX, and 5 pmole of each primer was prepared. Thermal cycling conditions consisted of one cycle of $30 \mathrm{sec}$ at $95^{\circ} \mathrm{C}$ followed by 45 cycles of $5 \mathrm{sec}$ at $95^{\circ} \mathrm{C}, 10 \mathrm{sec}$ at $55-61^{\circ} \mathrm{C}$, and $20 \mathrm{sec}$ at $72^{\circ} \mathrm{C}$. All qPCR experiments were repeated three times and relative quantification was performed by the $\Delta \Delta C T$ method. When mean genomic dosage ratios of the region between the target sample and female control DNA ( $\triangle \Delta \mathrm{CT}$ of target and internal control) were above two, the region was defined as a copy number gain.

\section{Association rule mining}

The association rule mining is used for finding interesting relations among variables in a database. In bioinformatics, the information metric was commonly used to assess the degree of "surprise" when a pattern actually occurs [23]. We used CPAR (Classification based on Predictive Association Rules) [24] algorithm adopting the information metric which was implemented by the LUCS-KDD research group (http://www.csc.liv.ac.uk/ $\sim$ frans/KDD/Software). In CPAR, Laplace accuracy is used to measure the accuracy of rules. Given a rule $r$, Laplace accuracy is defined as follows:

$$
\text { Laplace accuracy }(r)=\frac{\left(N_{c}+1\right)}{\left(N_{\text {total }}+m\right)}
$$

where $m$ is the number of classes and $N_{\text {total }}$ is the total number of examples that satisfy the rule's body, among which $N_{c}$ examples belong to the predicted class, C of the rule.

Through the CPAR algorithm, association rules were generated between RAR markers and the survival status in the discovery set. Each RAR marker was coded as 0 or 1 based on the copy number status; 0 indicates no copy number variation and 1 indicates copy number change in the marker region. Likewise, the survival status was coded as 0 (dead) or 1 (alive).

\section{Statistical analysis}

To examine the clinicopathologic implications of RARs, five clinical parameters were used as categorical variables: age at diagnosis (<50 vs. $\geq 50$ years), stage, ER status, PR status, and HER2 status. Differential distributions of RARs in each category were tested by a two-sided Fisher's exact test. The false discovery rate (FDR) was used for multiple comparison correction. In univariate survival analysis, cumulative overall survival was calculated according to the Kaplan-Meier method. Differences in survival curves were assessed with the log-rank test. Cox regression was performed to identify RARs associated with prognosis after 
adjusting for age, stage, ER, PR, and HER2. SAS version 9.1 (SAS Institute Inc., NC) was used and $P$-values less than 0.05 were considered significant in all statistical analyses.

\section{Results}

General characteristics of copy number alterations in EBC Genome-wide CNAs of the 48 EBCs (discovery set) were examined individually, as described in the Methods (Figure 1A). The median number of CNAs per each sample was 10 (range 1-21). Frequency plots of CNAs in the discovery set showed that alterations were not randomly distributed, but were clustered in several hot regions across the whole chromosomes (Figures $1 \mathrm{~A}$ and B). The overall CNA frequency profiles were similar for stage I and II groups, and among the molecular subtypes (Additional file 1: Figure S1). Of all 4,396 CNAs, copy number gains on $16 \mathrm{p} 13.3,16 \mathrm{p} 12.3$, and $17 \mathrm{q} 25$, and copy number losses on 16q21, 17p12, 17p13, and 20q11.1 were significantly more frequent in the stage II groups based on unadjusted $P$ values $(P<0.05)$, but none after multiple comparison correction (Additional file 1: Table $\mathrm{S} 2)$. On average, $376 \mathrm{Mb}$ (13\% of the whole genome) per $\mathrm{EBC}$ tissue specimen showed chromosomal alterations.

\section{Recurrently altered chromosomal regions in EBC}

Except for a few entire chromosomal arm changes, the majority of copy number alterations were regional changes, and some of them were observed repeatedly in the discovery set. Chromosomal segments that covered overlapping CNAs appeared in at least $30 \%$ of the samples with $P<0.05$ and were defined as RARs (RAR-G for gains and RAR-L for losses, respectively). A total of 23 RARs (18 RAR-Gs and 5 RAR-Ls) were defined in the discovery set (Table 2). Figure 2 illustrates the RAR on 17q12 as an example. Of the 23 RARs, 15 RARs detected commonly in both stage I and II groups can be considered earlier events in breast tumorigenesis. Two RARs (RAR-G14 and -G15), which appeared in less than $10 \%$ of the stage I samples but in over $40 \%$ of the stage II samples, appear to be relatively later events (Table 2). There were six RARs observed in over $50 \%$ of the discovery samples: RAR-G2 (1q21.2-q21.3), RAR-G7 (8q24.13), RAR-G8 (8q24.13-21), RAR-G9 (8q24.3), RAR-G10 (8q24.3), and RAR-L1 (8p23.1-p22). All six highly common RARs were earlier events, as described above. Their high prevalence and early occurrence suggest that potential driver cancer genes may be included within the segments. Some cancer-related genes are located in these highly common, early appearing RARs: MCL1, CTSK, ARNT, S100A10, PTK2, PTP4A3, and PSCA in the RAR-Gs; and DLC1, PINX1, and GATA4 in the RAR-Ls. Many known or putative cancer-related genes are also located in other RARs. For example, the $E R B B 2$, GRB2, and MMP families, as well as the TPM3, PYGO2, CKS1B, MUC1, CCT3, PRCC, UBE2C, and

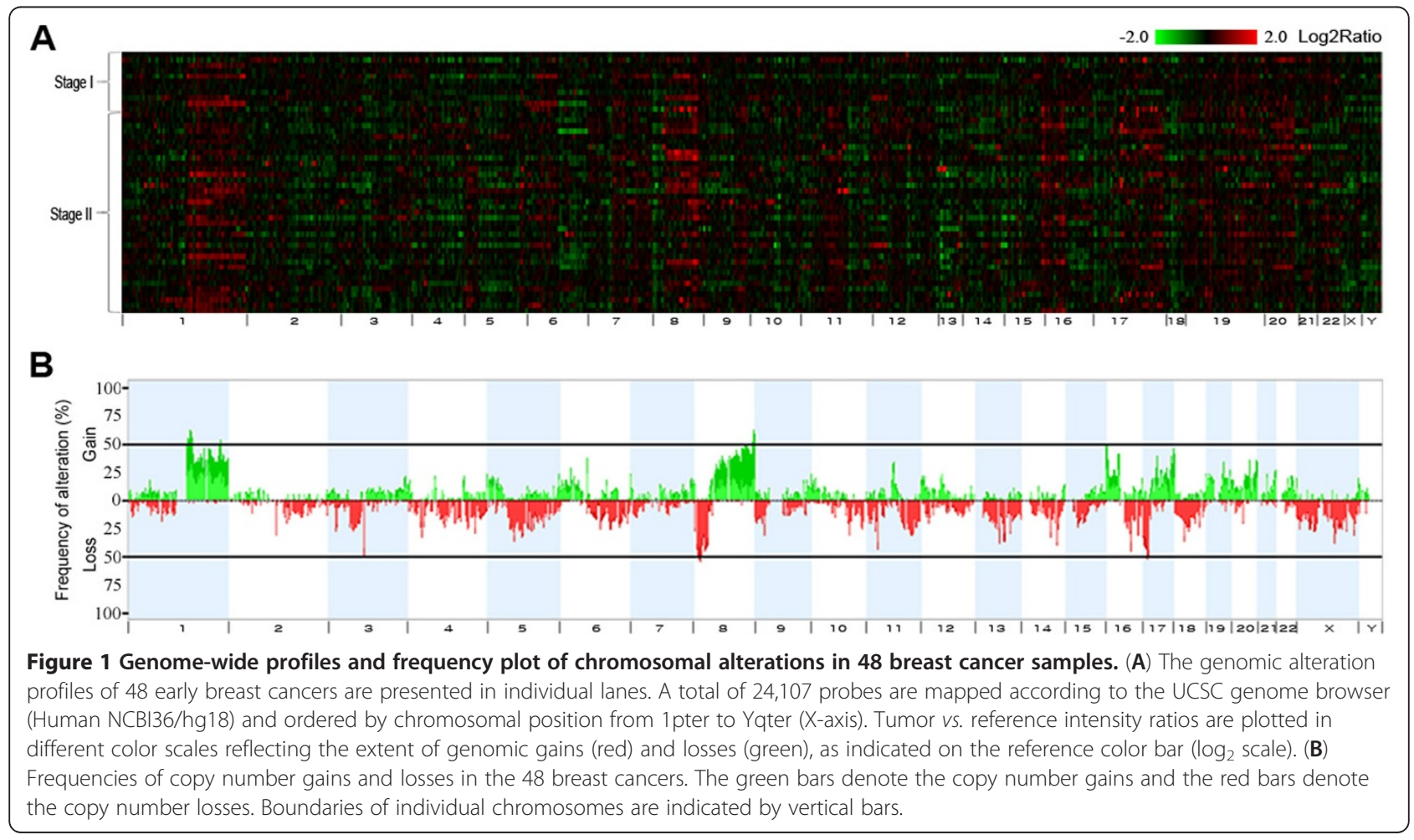


Table 2 Recurrently altered regions in $\mathbf{4 8}$ breast cancer specimens

\begin{tabular}{|c|c|c|c|c|c|c|c|c|c|c|}
\hline RARs & Chr & $\begin{array}{l}\text { Map position } \\
(\mathrm{Mp})\end{array}$ & $\begin{array}{l}\text { Size } \\
(\mathrm{Mp})\end{array}$ & Cytoband & $P$ - value & $\begin{array}{l}\text { Freq } \\
\text { (Total) }\end{array}$ & $\begin{array}{l}\text { Freq } \\
(\mathrm{S}-\mathrm{I})\end{array}$ & $\begin{array}{l}\text { Freq } \\
\text { (S-II) }\end{array}$ & Event* & Cancer-related genes \\
\hline RAR-G1 & 1 & $143,83-144,30$ & 0.47 & q21.1 & 0.04 & $48 \%$ & $36 \%$ & $51 \%$ & Earlier & TXNIP \\
\hline RAR-G2 & 1 & $148,12-150,25$ & 2.13 & $q 21.2-q 21.3$ & 0.01 & $50 \%$ & $36 \%$ & $54 \%$ & Earlier & MCL1, CTSK, ARNT, SETDB1, SELENBP1, S100A10 \\
\hline RAR-G3 & 1 & $150,75-155,11$ & 4.36 & q21.3-q23.1 & 0.04 & $48 \%$ & $36 \%$ & $51 \%$ & Earlier & $\begin{array}{l}\text { CREB3L4, TPM3, HAX1, IL6R, PYGO2, SHC1, } \\
\text { CKS1B, NES, ADAM15, EFNA1, MUC1, YY1AP1, } \\
\text { ARHGEF2, RAB25, CCT3, CRABP2, HDGF, PRCC }\end{array}$ \\
\hline RAR-G4 & 1 & $158,48-159,47$ & 0.99 & q23.2-q23.3 & 0.04 & $48 \%$ & $45 \%$ & $49 \%$ & Earlier & F11R, USF1 \\
\hline RAR-G5 & 1 & $226,10-226,90$ & 0.80 & $q 24.13$ & 0.04 & $48 \%$ & $45 \%$ & $49 \%$ & Earlier & WNT9A, WNT3A \\
\hline RAR-G6 & 6 & $64,05-64,75$ & 0.70 & $\mathrm{q} 12$ & 0.00 & $38 \%$ & $45 \%$ & $35 \%$ & Earlier & - \\
\hline RAR-G7 & 8 & $123,47-124,74$ & 1.27 & $q 24.13$ & 0.02 & $50 \%$ & $36 \%$ & $54 \%$ & Earlier & FAM83A \\
\hline RAR-G8 & 8 & $126,23-127,30$ & 1.07 & $q 24.13-q 24.21$ & 0.02 & $50 \%$ & $36 \%$ & $54 \%$ & Earlier & - \\
\hline RAR-G9 & 8 & $140,69-143,86$ & 3.17 & q24.3 & 0.02 & $50 \%$ & $36 \%$ & $54 \%$ & Earlier & PTK2, PTP4A3, PSCA, LYNX1 \\
\hline RAR-G10 & 8 & $144,34-146,27$ & 1.93 & q24.3 & 0.02 & $50 \%$ & $36 \%$ & $54 \%$ & Earlier & SCRIB, HSF1, SLCA4 \\
\hline RAR-G11 & 16 & $0-3,22$ & 3.22 & p13.3 & 0.05 & $38 \%$ & $27 \%$ & $41 \%$ & $U C$ & $\begin{array}{l}\text { MPG, AXIN1, RHOT2, STUB1, MSLN, CACNA1H, } \\
\text { IGFALS, GFER, TSC2, PDPK1, MMP25 }\end{array}$ \\
\hline RAR-G12 & 16 & $28,32-31,19$ & 2.87 & p11.2 & 0.05 & $40 \%$ & $36 \%$ & $41 \%$ & Earlier & $\begin{array}{l}\text { NUPR1, SULT1A2, SULT1A1, MVP, MAPK3, } \\
\text { FUS, PYCARD }\end{array}$ \\
\hline RAR-G13 & 17 & $34,97-35,30$ & 0.33 & $\mathrm{q} 12$ & 0.03 & $38 \%$ & $36 \%$ & $38 \%$ & Earlier & PPP1R1B, PNMT, PERLD1, ERBB2, GRB7 \\
\hline RAR-G14 & 17 & $70,76-71,21$ & 0.45 & q25.1 & 0.03 & $38 \%$ & $9 \%$ & $46 \%$ & Later & GRB2 \\
\hline RAR-G15 & 17 & $76,90-78,77$ & 1.87 & q25.3 & 0.03 & $38 \%$ & $9 \%$ & $46 \%$ & Later & HGS, SIRT7, RAC3, FASN, CSNK1D \\
\hline RAR-G16 & 20 & $43,84-44,15$ & 0.31 & $q 13.12$ & 0.00 & $33 \%$ & $18 \%$ & $38 \%$ & UC & UBE2C, MMP9 \\
\hline RAR-G17 & 20 & $60,17-61,61$ & 1.44 & q13.33 & 0.04 & $31 \%$ & $18 \%$ & $35 \%$ & UC & ADRM1, LAMA5, NTSR1, BIRC7, EEF1A2 \\
\hline RAR-G18 & 20 & $61,67-62,44$ & 0.77 & q13.33 & 0.04 & $31 \%$ & $27 \%$ & $32 \%$ & $U C^{*}$ & TNFRSF6B \\
\hline RAR-L1 & 8 & $8,14-14,07$ & 5.93 & p23.1-p22 & 0.00 & $50 \%$ & $36 \%$ & $54 \%$ & Earlier & CLDN23, PINX1, GATA4, NEIL2, FDFT1, CTSB, DLC1 \\
\hline RAR-L2 & 8 & $15,66-17,12$ & 1.44 & $\mathrm{p} 22$ & 0.02 & $48 \%$ & $36 \%$ & $51 \%$ & Earlier & TUSC3, MSR1, FGF20 \\
\hline RAR-L3 & 8 & $24,01-26,72$ & 2.71 & p21.2 & 0.02 & $48 \%$ & $36 \%$ & $51 \%$ & Earlier & ADAM28, GNRH1, PPP2R2A, ADRA1A \\
\hline RAR-L4 & 16 & $75,79-80,74$ & 4.95 & $\mathrm{q} 23.1-\mathrm{q} 23.3$ & 0.00 & $33 \%$ & $27 \%$ & $35 \%$ & $U C$ & WWOX, DYNLRB2, PLCG2, HSD17B2 \\
\hline RAR-L5 & 17 & $10,54-14,10$ & 3.56 & p13.1-p12 & 0.04 & $44 \%$ & $27 \%$ & $49 \%$ & UC & MAP2K4, ELAC2 \\
\hline
\end{tabular}

RAR-G: RAR-gain, RAR-L: RAR-loss, Chr: chromosome, Freq: frequency, S-I: stage I, S-II: stage II.

Cancer-related genes: Among all RefSeq genes located in the RARs, the genes which can be searched by the keyword "Cancer/Tumor" from NCBI Gene (http://www.ncbi.nlm.nih.gov/gene) are listed as "cancer-related genes".

*Earlier: RARs detected commonly in both stage I and II groups, Later: RARs appearing in $<10 \%$ of stage I samples, but in $>40 \%$ of stage II samples, UC: unclassified.

TNFRSF6 $B$ genes are located in the RAR-Gs while the PPP2R2A, WWOX, TUSC3, MAP2K4, and ELAC2 genes are located in the RAR-Ls (Table 2).

\section{High-level CNAs in EBC}

Of the CNAs identified in our EBC specimens, 191 loci were determined to be high-level CNAs (defined as $\geq 1.5$ for amplification or $\leq-1.5$ for HD on the $\log _{2}$ scale), with 158 amplifications and 33 HDs (Additional file 1: Table S3). Of the 158 amplifications, 5 were detected in over $10 \%$ of the samples: 1q21.3, 8q24.22, 8q24.3, 16p11.2, and 17q12 (Table 3). These five relatively common amplifications overlapped with earlier event RAR-Gs. Amplification on 17q12, where the EBBB2 oncogene is located, was the most frequent occurrence (14/48, 29\%), followed by amplification on 1q21.3 and 8q24.22 (both 9/
48, 19\%), where the $L C E$ families and $M Y C$ gene are located, respectively. Examples of high copy number changes are illustrated in Figure 3. There was no HD detected in over $10 \%$ of the samples.

\section{Association of RARs with clinicopathologic features}

Five clinical variables (age at diagnosis, stage, ER status, PR status, and HER2 status) were analyzed to assess their association with RARs (see Additional file 1). Only RAR-L4 was significantly associated with ER-positivity $(P<0.0001$, FDR-corrected $\mathrm{P}=0.015)$ (Additional file 1: Table S4). When we observed the distribution of the RARs by molecular subtypes (Luminal A, Luminal B, HER2 and TNBC), only RAR-G13 was differently distributed among the subtypes $\left(P=1.77 \times 10^{-4}\right)$ (Additional file 1: Table S5). 


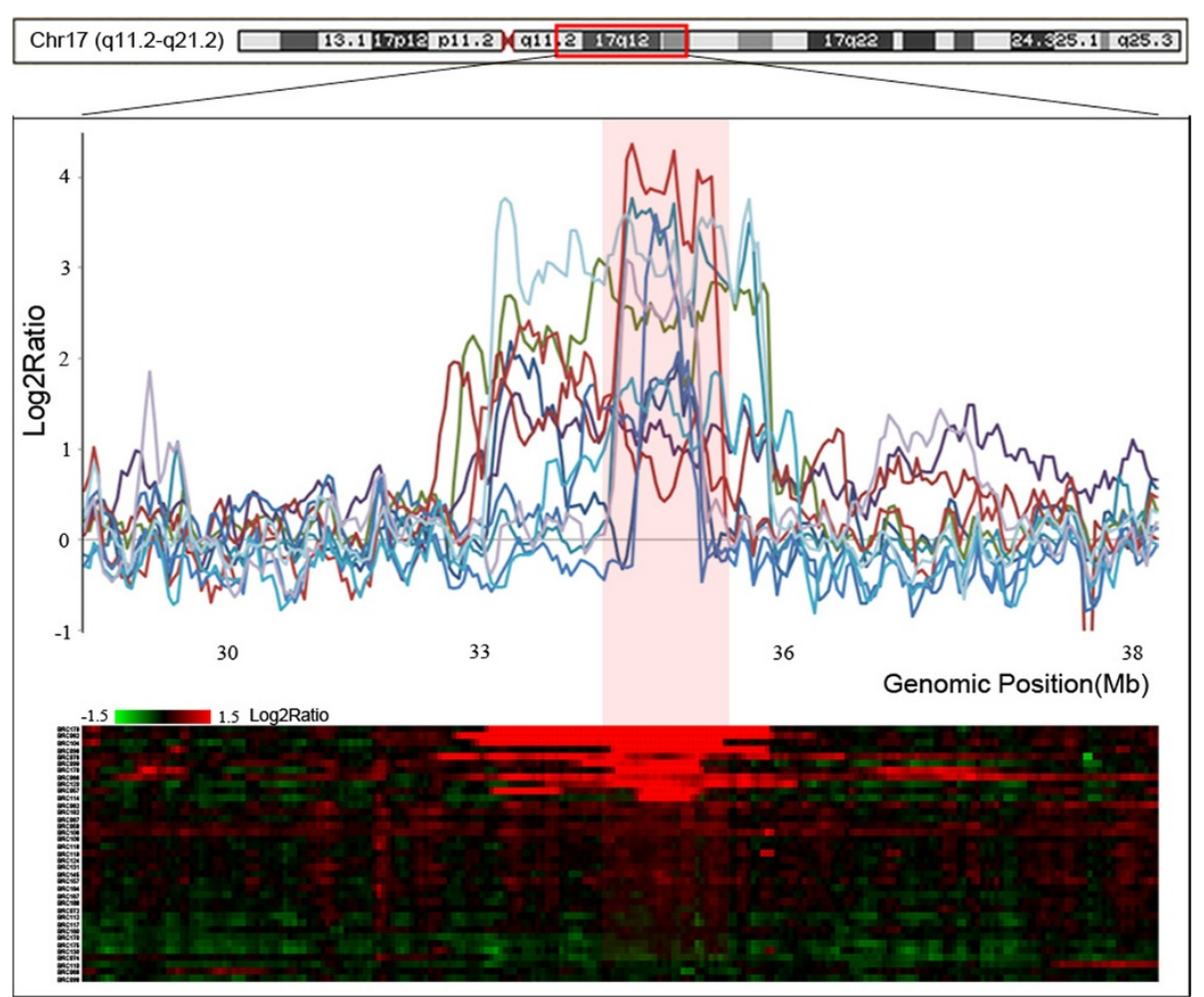

Figure 2 Example of a recurrently altered region on 17q12. The bottom plot represents the copy number profile of 48 breast cancers around $7 q 12$ as a heat map. Intensity ratios are plotted in different color scales, reflecting the extent of genomic gains (red) and losses (green), as indicated on the reference color bar. The upper plot illustrates examples of intensity ratios of individual breast cancers. The red zone represents RARs defined using our criteria (frequency $>30 \%$ and $P<0.05$; NEXUS software v3.1). X-axis, genomic position (Mb); Y-axis, Log 2 Ratio.

\section{RARs associated with prognosis in EBC}

Univariate survival analysis was performed to screen RARs that have potential implications on patient survival. Univariate analysis was also performed to identify clinicopathologic features (age at diagnosis, stage, ER, $\mathrm{PR}$, and HER2) for inclusion as covariates for Cox regression. The event was defined as a death within ten years of the diagnosis. Among the genetic and clinical factors, RAR-G12 (16p11.2) and RAR-G13 (17q12) were significantly associated with poor survival in the discovery set of $48 \mathrm{EBCs}(P=0.0186$ and $P=0.0186$, respectively) (Figures $4 \mathrm{~A}$ and $\mathrm{B}$ ).

The role of combinations of the 23 RARs and their association with poor survival was also explored, as described in the Methods. As a result, eight combination rules were found to be potentially associated with death events (Laplace accuracy score >0.75) (Additional file 1: Table S6). All eight poor survivalassociated rules contained 'RAR-G12 and RAR-G13 positives', suggesting that the co-occurrence of these two alterations may affect EBC prognosis. Indeed, on univariate survival analysis, RAR-G12 and -13 doublepositives had a significantly worse prognosis compared with the prognosis of others $(P=0.0001)$ (Figure $4 C$ ).
Among the clinicopathologic features, none of them was associated with survival.

\section{Replication of the poor prognosis-associated RARs}

In order to validate the prognosis-associated RARs identified in the discovery set, a genomic qPCR system was designed to target the NUPR1 gene located in RAR-G12 and the ERBB2 gene located in RAR-G13 (Figure 5A). For independent validation, the DNA extracted from FFPE samples of 97 EBCs (replication set) was used. On univariate survival analysis of the replication set, patients positive for RAR-G13 (ERBB2) were found to have a significantly poorer survival than patients without the $E R B B 2$ gain $(P=0.0038)$ (Figure $5 \mathrm{~B})$. Patients positive for RAR-G12 (NUPR1) also showed poorer survival than negative patients, but only with borderline significance $(P=0.0839)$ (Figure $5 C)$. As expected, the patients positive for both NUPR1 and ERBB2 gains had a significantly poorer prognosis than patients in the replication set that did not have this combination $(P=0.0014)$ (Figure 5D). When survival curves were compared based on a more detailed RAR status (positive for both RAR-G12 and -13 , positive for either RAR-G12 or -13 , and negative for both), the survival probabilities among the three 
Table 3 Common high-copy number changes ( $>10 \%$ )

\begin{tabular}{|c|c|c|c|c|c|c|c|c|}
\hline Changes & Chr & Position (Mb) & Cytoband & Size (Mb) & Frequency & DGV $^{a}$ & Korean $\mathrm{CNV}^{\mathrm{b}}$ & Putative cancer-related genes \\
\hline AMP9 & 1 & 150.82-150.93 & $1 \mathrm{q} 21.3$ & 0.11 & $9(19 \%)$ & Y & Y & LCE3C, LCE3B, LCE3A, LCE2D, LCE2C, LCE2B \\
\hline AMP64 & 8 & $120.51-135-87$ & $8 q 24.22$ & 15.37 & $9(19 \%)$ & Y & Y & $\begin{array}{l}\text { DDEF1, ENPP2, FAM83A, FAM84B, } \\
\text { HAS2, MTSS1, MYC, NDRG1, RNF139, TG, WISP1 }\end{array}$ \\
\hline AMP67 & 8 & 144.37-145.55 & $8 q 24.3$ & 1.18 & $5(10 \%)$ & Y & Y & SCRIB, PLEC1, HSF1 \\
\hline AMP113 & 16 & $28.40-28.42$ & $16 p 11.2$ & 0.02 & $6(13 \%)$ & Y & N & CLN3 \\
\hline AMP119 & 17 & $32.42-35.87$ & $17 q 12$ & 3.45 & $14(29 \%)$ & Y & $\mathrm{N}$ & $\begin{array}{l}\text { AATF, ACACA, CDC6, CSF3, ERBB2, FBXO47, } \\
\text { GRB7, IGFBP4, LASP1, PCGF2, PERLD1, PLXDC1, } \\
\text { PNMT, PPP1R1B, RARA, RPL19, RPL23, TBC1D3, } \\
\text { THRA, TOP2A }\end{array}$ \\
\hline
\end{tabular}

${ }^{a}$ Overlapping status with DGV (http://projects.tcag.ca/variation/) entries; ${ }^{\mathrm{b}}$ Overlapping status with CNVs identified from Koreans [35].

Y: overlap, N: no overlap, AMP: amplification, Chr: chromosome.

groups were significantly different, with the probability of surviving decreasing with greater RAR positivity $(P=0.0052 ; P$ for trend $=0.0020)$ (Figure $5 \mathrm{E})$.

Multivariate analysis with the two significant RARs identified on univariate analysis and the covariates (age at diagnosis, stage, ER status, PR status, and HER2 status) revealed that the copy number gain status of ERBB2 (RAR-G13) was an independent indicator of poor prognosis in EBC (Hazard ratio $[\mathrm{HR}]=5.36,95 \% \mathrm{CI}$ 1.80-15.98, $P=0.003$ ) (Table 4 ). When the multivariate analysis was performed with NUPR1-ERBB2 combined status and the same covariates, positivity for both RAR-
Gs was found to be a strong independent indicator of poor prognosis, showing additive effects $(\mathrm{HR}=7.31$, 95\% CI 2.65-20.15, $P=0.0001$ ) (Table 4).

\section{Discussion}

In this study, we analyzed the genome-wide copy number alteration profiles in 48 EBCs using 30K oligoarray$\mathrm{CGH}$. We delineated RARs under the assumption that commonly altered chromosomal segments in EBCs may contain driver genes essential for initiation or early progression of breast tumorigenesis. It is also possible that some RARs have prognostic implications in EBC. To

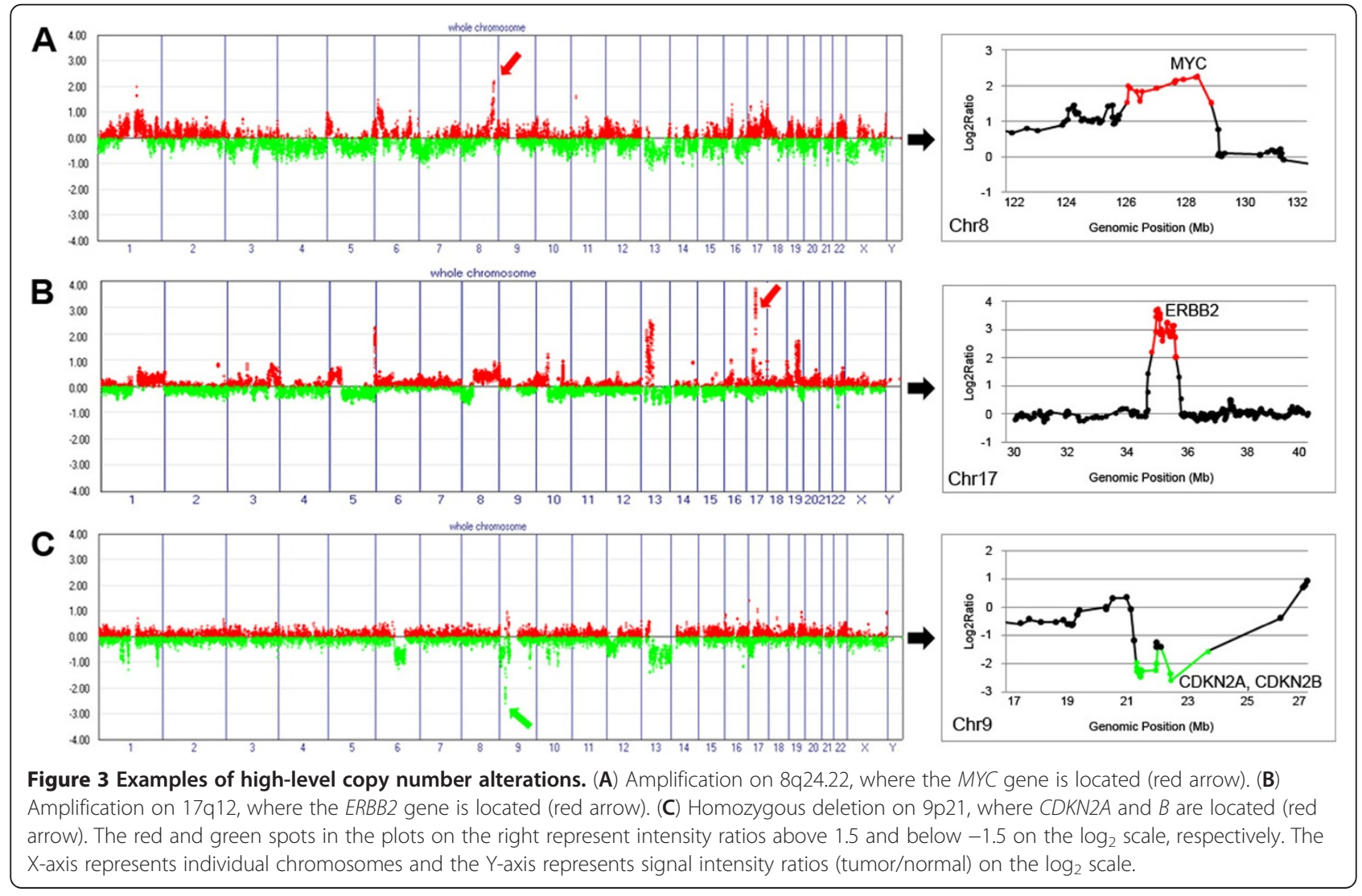



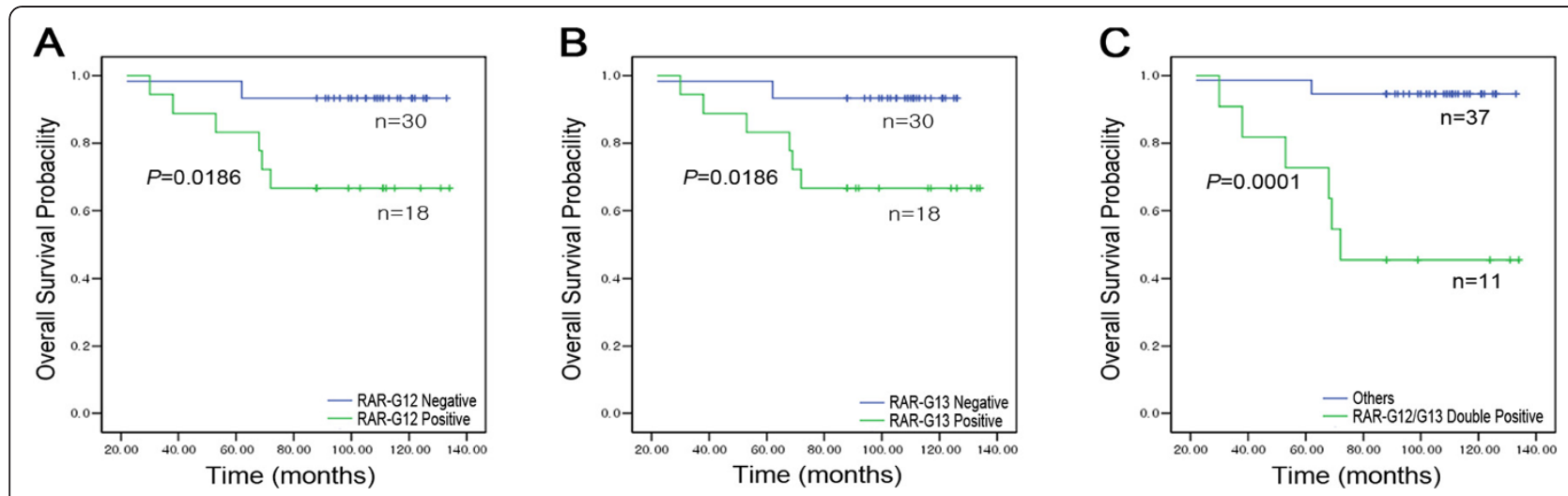

Figure 4 Kaplan-Meier curves by RAR status in the discovery set. (A) RAR-G12 (16p11.2) positives vs. negatives, (B) RAR-G13 (17q12) positives vs. negatives, and (C) RAR-G12/G13 double-positives vs. others. The curves show overall survival.

explore this possibility, we defined RARs in a discovery set of EBC and examined their associations with prognosis. A total of 23 RARs were defined, and all of them were found to overlap at least one of the recently reported CNAs in breast cancer including EBC, suggesting the reliability of our data [14-17]. The nature of RARs (gain or loss) was also largely consistent with the previous observations. For example, RAR-L3 (8p21.2) and RAR-L5 (17p12), where PPP2R2A and MAP2K4 are located, respectively, and RAR-G13 (17q12), where $E R B B 2$ is located, were consistently detected in a recent large-scale breast cancer genetic subgroup study [14]. In particular, 21 of the 23 RARs overlap recurrent copy number alterations identified in EBCs (stage I and II) from whites, blacks, and Hispanics by Thompson et al.'s recent study [15]. However, the recurrent gain on 14q11.2 in Thompson et al.'s report was not detected in our array-CGH analysis. This difference, which requires further investigation, may be due to a Korean EBCspecific feature or to the probe design of the arrayplatform used in this study. We validated the association of RARs with prognosis in the larger independent replication set of 97 EBCs. In addition to RARs, some entire chromosomal arm changes were also commonly observed (> 30\% of the samples) in this study (Additional file 1: Table S7), and are largely consistent with previous observations in breast cancer of diverse ethnic groups $[11,25]$.

Of the RARs identified in this study, 15 were commonly detected in both stages I and II, which suggests

\section{A

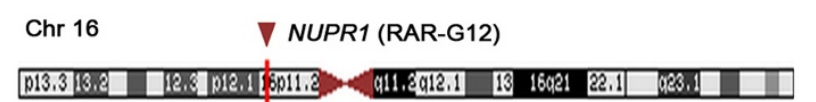

Chr 17

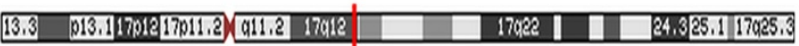
ए Validation of prognostic implication of the two RARs by genomic qPCR

B

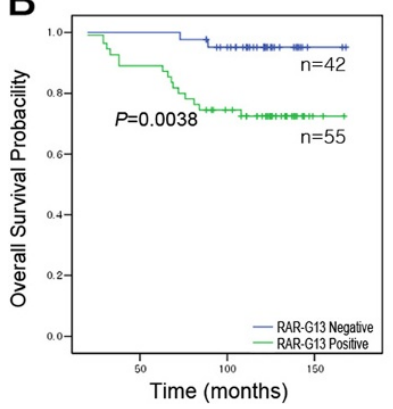

C

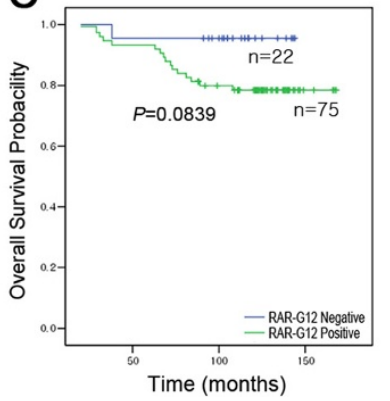

D

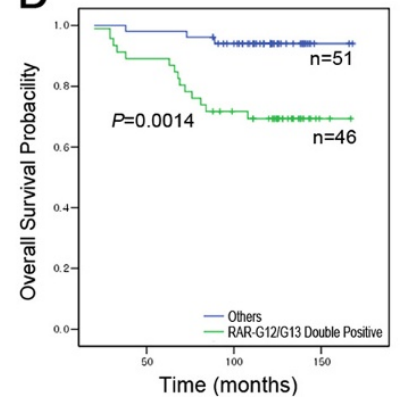

$\mathrm{E}$

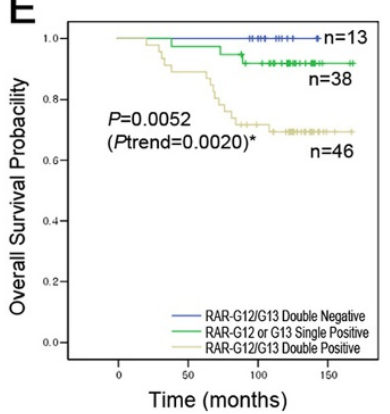

Figure 5 Kaplan-Meier curves by RAR status in the replication set. (A) Genomic QPCR system targets; NUPR1 gene located in RAR-G12; ERBB2 gene located in RAR-G13. (B) NUPR1 (RAR-G12) positives vs. negatives. (C) ERBB2 (RAR-G13) positives vs. negatives. (D) NUPR1-ERBB2 double-positives vs. others. (E) Survival curves by subgroup; RAR-G12/13 double-positives, positive for either RAR-G12 or G-13, and double-negatives. The curves show overall survival. 
Table 4 Results of multivariate aalysis

\begin{tabular}{|c|c|c|c|c|}
\hline \multirow[t]{2}{*}{ Variable } & \multirow[t]{2}{*}{ Hazard ratio } & \multicolumn{2}{|c|}{ 95\% confidence interval } & \multirow[t]{2}{*}{$P$ value* } \\
\hline & & Lower & Upper & \\
\hline \multicolumn{5}{|l|}{ Using each RAR } \\
\hline Age & 1.01 & 0.41 & 2.48 & 0.986 \\
\hline Stage & 3.54 & 1.01 & 12.39 & 0.048 \\
\hline ER & 1.63 & 0.61 & 4.34 & 0.325 \\
\hline PR & 0.82 & 0.32 & 2.13 & 0.690 \\
\hline HER2 & 0.48 & 0.19 & 1.17 & 0.105 \\
\hline RAR-G12 & 2.55 & 0.73 & 8.90 & 0.141 \\
\hline RAR-G13 & 5.36 & 1.80 & 15.98 & 0.003 \\
\hline \multicolumn{5}{|c|}{ Using RAR double-positives } \\
\hline Age & 1.00 & 0.41 & 2.44 & 0.993 \\
\hline Stage & 3.73 & 1.06 & 13.04 & 0.040 \\
\hline ER & 1.60 & 0.61 & 4.24 & 0.343 \\
\hline PR & 0.84 & 0.32 & 2.15 & 0.709 \\
\hline HER2 & 0.46 & 0.19 & 1.14 & 0.094 \\
\hline RAR-G12*RAR-G13 & 7.31 & 2.65 & 20.15 & 0.0001 \\
\hline
\end{tabular}

RAR-G12*RAR-G13: NUPR1 (RAR-G12) and ERBB2 (RAR-G13) copy number gain double-positives.

*Significant result $(P<0.05)$.

that these copy number alterations were acquired at an earlier stage of EBC. In particular, 6 of the 15 earlier event RARs, RAR-G2 (1q21.2-q21.3), RAR-G7 (8q24.13), RAR-G8 (8q24.13-21), RAR-G9 (8q24.3), RAR-G10 (8q24.3), and RAR-L1 (8p23.1-p22), appeared in over $50 \%$ of cases. Some genes located in these six RARs have been suggested to be involved in early breast tumorigenesis. For instance, the PTK2 gene located on $8 \mathrm{q} 24.3$ (RAR-G9) is a member of the focal adhesion kinase (FAK) subfamily of protein tyrosine kinases. Overexpression of FAK was suggested to be an early event in DCIS tumorigenesis [26]. Although the protein levels of potential cancer-related genes in these six highly common loci were not examined in this study, our data suggest that the six alterations may be commonly occurring genetic events in the initial stage of breast cancer development. Based on our findings, two RARs on 17q25 can be considered relatively late events in breast tumorigenesis, since the RARs on 17q25 (RAR-G14 and -G15) were scarcely observed in stage I $(<10 \%)$, but were quite frequent $(>45 \%)$ in stage II. Interestingly, a copy number gain on $17 \mathrm{q} 25.3$ was reported to be one of the recurrence-associated chromosomal alterations in one previous report on Korean women with breast cancer [27].

When we assessed the prognostic implications of RARs, RAR-G12 (16p11.2) and RAR-G13 (17q12) were significantly associated with poorer prognosis in the discovery set. A number of cancer-related genes are located in these two RARs: NUPR1, MVP, MAPK3, FUS, and
PYCARD are located in RAR-G12 while ERBB2, GRB7, and PPP1R1B are located in RAR-G13. Among these potential cancer-related genes, Nupr1 is known to interact with various molecules involved in cell cycle regulation, programmed cell death and transcription activity. For these reasons, Nupr1 is a potential molecular target in the development of anticancer drugs [28]. Although the NUPR1 gene has been suggested to be responsible for the growth and progression of many cancers including breast cancer [29,30], the prognostic implications of the NUPR1 gene in EBC have not been reported. Amplification and overexpression of the ERBB2 oncogene in RAR-G13 (17q12) is known to be associated with high recurrence rates and reduced breast cancer survival [31-33]. The frequent copy number gains (38\%) and amplification (29\%) of ERBB2 in this study are consistent with previous studies on breast cancer $[11,34]$.

In a replication analysis by genomic qPCR, the prognostic implication of ERBB2 gain (RAR-G13) was successfully replicated in the larger replication set, but that of the NUPR1 gain (RAR-G12) was not. We hypothesized that the NUPR1 gain itself might not be an influential alteration, but that EBC prognosis is more strongly affected by the co-occurrence of NUPR1 with a strong driver mutation (ERBB2). Association-rule mining results also supported the predictive power of their cooccurrence for poor prognosis. As expected, when these two RARs were combined and used as an independent factor, the hazard ratio increased in an additive manner. A stronger significance level was also achieved on Cox regression analysis compared with when only ERBB2 was used, which may reflect the multigenic nature of cancer.

In this study, 191 high-level CNAs (158 amplifications and $33 \mathrm{HDs}$ ) were detected by array-CGH, and 5 of them were detected in more than $10 \%$ of the samples. A substantial number of the high-level CNAs overlap database of genomic variants (DGV, http://projects.tcag.ca/ variation/) entries and the copy number variants (CNVs) identified from Koreans [35]. Although the limitations of DGV are well known in terms of accuracy and overestimation, we cannot rule out the possibility that some high-level CNAs identified in this study are copy CNVs because we used DNA from a single individual as a universal reference. All five of the common amplifications (observed in $>10 \%$ of the samples) also overlap the CNV loci in DGV. However, four of them, except for one very small $(0.02 \mathrm{Mb})$ amplification on 16p11.2, were reported to be amplifications or copy number gains in breast cancer by a recent high-resolution array-CGH analysis $[15-17,36]$, suggesting that these four common amplifications are likely CNAs. The amplification frequency of $E R B B 2$ in this study was largely similar to the previous studies including Koreans [37-39]. 
There are several limitations in this study. First, due to the limited sample size of subtypes, we could not see the prognostic implications of the RARs in the four molecular subtypes properly. Second, we did not examine the molecular mechanisms of the synergistic effect of the ERBB2-NUPR1 co-occurrence. Further studies will be required to delineate the roles of NUPR1 gain and the simultaneous ERBB2-NUPR1 gains in early breast tumorigenesis. Third, we used single reference DNA in this study, so it is possible that some of the CNAs identified in this study are CNVs, especially smallsized CNAs overlapping previously reported CNVs.

\section{Conclusion}

In this study, we found six highly common RARs in EBCs and determined the potential of simultaneous alterations of ERBB2 (17q12) and NUPR1 (16p11.2) as significant predictors of poor prognosis in EBC. Our study will help to elucidate the molecular mechanisms underlying early-stage tumorigenesis in breast cancer. In addition, our study shows the potential for combinations of copy number alterations to be used as prognosis predictors for early-stage breast cancer.

\section{Additional file}

Additional file 1: Figure S1. Genome-wide frequency plots of chromosomal alterations for each breast cancer subtype. Table S1. Primer sequences for target and diploid control regions. Table S2. Copy number alterations significantly more frequent in stage II than in stage I. Table S3. High copy number changes in the discovery set of early breast cancers. Table S4. Correlation between RARs and clinicopathologic features. Table S5. RARs in 48 breast cancers by molecular subtype. Table S6. RAR combination rules associated with death events. Table S7. Frequency of chromosomal arm changes in 48 breast cancers.

\section{Abbreviations}

EBC: Early-stage breast cancer; CNA: Copy number alteration; RAR: Recurrently altered region; array-CGH: Oligoarray-comparative genomic hybridization.

\section{Competing interests}

The authors declare that they have no competing interests.

\section{Authors' contribution}

SHJ executed most experiments and drafted the manuscript. AWL collected the patient specimens and was involved in data analysis. SHY participated in the design of this study, performed statistical analysis and wrote the manuscript. HJH performed an association rule mining and drafted the manuscript. CC was involved in data analysis and preparing the figures. YJC proposed this study, organized the research team, interpreted all the data, and participated in writing the manuscript. All authors read and approved the final manuscript.

\section{Acknowledgements}

This study was supported by a grant from the Korea Healthcare technology R\&D Project (A092258), Korea Health 21 R\&D Project (A040002) and MRC for Cancer Evolution Research Center (2012047939), Republic of Korea. The authors wish to thank Dr HH Song for statistical comments.

\section{Author details}

'Integrated Research Center for Genome Polymorphism, College of Medicine, The Catholic University of Korea, 505 Banpo-dong, Socho-gu, Seoul 137-701, Republic of Korea. ${ }^{2}$ Department of Microbiology, The Catholic University of Korea, Seoul, South Korea. ${ }^{3}$ Department of Hospital Pathology, Humanities and Social Sciences, The Catholic University of Korea, Seoul, South Korea. ${ }^{4}$ Department of Medical Humanities and Social Sciences, The Catholic University of Korea, School of Medicine, 505 Banpo-dong, Seocho-gu, Seoul 137-701, South Korea.

Received: 13 November 2011 Accepted: 9 August 2012

Published: 31 August 2012

\section{References}

1. Jemal A, Siegel R, Ward E, Murray T, Xu J, Thun MJ: Cancer statistics. CA Cancer J Clin 2007, 57:43-66.

2. Hortobagyi GN, de la Garza Salazar J, Pritchard K, Amadori D, Haidinger R, Hudis CA, Khaled H, Liu MC, Martin M, Namer M, O'Shaughnessy JA, Shen ZZ, Albain KS, ABREAST Investigators: The global breast cancer burden: variations in epidemiology and survival. Clin Breast Cancer 2005, 6:391-401.

3. Beral V, Hermon C, Reeves G, Peto R: Sudden fall in breast cancer death rates in England and Wales. Lancet 1995, 345:1642-1643.

4. Jatoi I, Miller AB: Why is breast-cancer mortality declining? Lancet Oncol 2003, 4:251-254.

5. Rosner D, Lane WW: Predicting recurrence in axillary-node negative breast cancer patients. Breast Cancer Res Treat 1993, 25:127-139.

6. Rosen PR, Groshen S, Saigo PE, Kinne DW, Hellman S: A long-term followup study of survival in stage I (T1NOMO) and stage II (T1N1M0) breast carcinoma. J Clin Oncol 1989, 7:355-366.

7. Mirza AN, Mirza NQ, Vlastos G, Singletary SE: Prognostic factors in nodenegative breast cancer: a review of studies with sample size more than 200 and follow-up more than 5 years. Ann Surg 2002, 235:10-26.

8. American Cancer Society. http://www.cancer.org/cancer/breastcancer/ overviewquide/breast-cancer-overview-survival-rates.

9. Balmain A, Gray J, Ponder B: The genetics and genomics of cancer. Nat Genet 2003, 33:238-244.

10. Kwei KA, Kung Y, Salari K, Holcomb IN, Pollack JR: Genomic instability in breast cancer: pathogenesis and clinical implications. Mol Oncol 2010, 4:255-266.

11. Tsuda H: Gene and chromosomal alterations in sporadic breast cancer: correlation with histopathological features and implications for genesis and progression. Breast Cancer 2009, 16:186-201.

12. Tsuda H, Hirohashi S: Multiple developmental pathways to highly aggressive breast cancers disclosed by comparison of histologic grades and c-erbB-2 expression patterns in both the intraductal and invasive portions. Pathol Int 1998, 48:518-525.

13. Riener MO, Nikolopoulos E, Herr A, Wild PJ, Hausmann M, Wiech T, Orlowska-Volk M, Lassmann S, Walch A, Werner M: Microarray comparative genomic hybridization analysis of tubular breast carcinoma shows recurrent loss of the CDH13 locus on 16q. Hum Pathol 2008, 39:1621-1629

14. Curtis C, Shah SP, Chin SF, Turashvili G, Rueda OM, Dunning MJ, Speed D, Lynch AG, Samarajiwa S, Yuan Y, Gräf S, Ha G, Haffari G, Bashashati A, Russell R, McKinney S, METABRIC Group, Caldas C, Aparicio S, Writing committee, Curtis C, Shah SP, Caldas C, Aparicio S, Steering committee, Brenton JD, Ellis I, Huntsman D, Pinder S, Purushotham A, et al: The genomic and transcriptomic architecture of 2,000 breast tumours reveals novel subgroups. Nature 2012, 486:346-352.

15. Thompson PA, Brewster AM, Kim-Anh D, Baladandayuthapani V, Broom BM, Edgerton ME, Hahn KM, Murray JL, Sahin A, Tsavachidis S, Wang Y, Zhang L, Hortobagyi GN, Mills GB, Bondy ML: Selective genomic copy number imbalances and probability of recurrence in early-stage breast cancer PLoS One 2011, 6:e23543

16. Jönsson G, Staaf J, Vallon-Christersson J, Ringnér $M$, Holm K, Hegardt C, Gunnarsson H, Fagerholm R, Strand C, Agnarsson BA, Kilpivaara O, Luts L, Heikkilä P, Aittomäki K, Blomqvist C, Loman N, Malmström P, Olsson H, Johannsson OT, Arason A, Nevanlinna H, Barkardottir RB, Borg A: Genomic subtypes of breast cancer identified by array-comparative genomic hybridization display distinct molecular and clinical characteristics. Breast Cancer Res 2010, 12:R42. 
17. Natrajan R, Weigelt B, Mackay A, Geyer FC, Grigoriadis A, Tan DS, Jones C, Lord CJ, Vatcheva R, Rodriguez-Pinilla SM, Palacios J, Ashworth A, Reis-Filho JS: An integrative genomic and transcriptomic analysis reveals molecular pathways and networks regulated by copy number aberrations in basal-like, HER2 and luminal cancers. Breast Cancer Res Treat 2010, 121:575-589.

18. Russnes HG, Vollan HK, Lingjaerde OC, Krasnitz A, Lundin P, Naume B, Sørlie

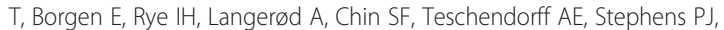
Månér S, Schlichting E, Baumbusch LO, Kåresen R, Stratton MP, Wigler M, Caldas C, Zetterberg A, Hicks J, Børresen-Dale AL: Genomic architecture characterizes tumor progression paths and fate in breast cancer patients. Sci Trans/ Med 2010, 2:38-47.

19. Perou CM, Sørlie T, Eisen MB, van de Rijn M, Jeffrey SS, Rees CA, Pollack JR, Ross DT, Johnsen H, Akslen LA, Fluge O, Pergamenschikov A, Williams C, Zhu SX, Lønning PE, Børresen-Dale AL, Brown PO, Botstein D: Molecular portraits of human breast tumours. Nature 2000, 406:747-752.

20. Jung SH, Shin SH, Yim SH, Choi HS, Lee SH, Chung YJ: Integrated analysis of copy number alteration and RNA expression profiles of cancer using a high-resolution whole-genome oligonucleotide array. Exp Mol Med 2009, 41:462-470

21. Kim SY, Nam SW, Lee SH, Park WS, Yoo NJ, Lee JY, Chung YJ: ArrayCyGHt: a web application for analysis and visualization of array-CGH data. Bioinformatics 2005, 21:2554-2555.

22. Yim SH, Chung YJ, Jin EH, Shim SC, Kim JY, Kim YS, Hu HJ, Shin SH, Pae HO, Zouali M, Chung HT: The potential role of VPREB1 gene copy number variation in susceptibility to rheumatoid arthritis. Mol Immunol 2011, 48:1338-1343.

23. Agrawal R, Imielinski T, Swami A: Database mining: A performance perspective. IEEE T KNOWL DATA EN 1993, 5:914-925

24. Yin X, Han J: CPAR: Classification based on predictive association rules. In: SIAM International Conference of Data Mining. San Fransisco, CA: SIAM; 2003:331-335.

25. van Beers EH, Nederlof PM: Array-CGH and breast cancer. Breast Cancer Res 2006, 8:210.

26. Lightfoot HM Jr, Lark A, Livasy CA, Moore DT, Cowan D, Dressler L, Craven RJ, Cance WG: Upregulation of focal adhesion kinase (FAK) expression in ductal carcinoma in situ (DCIS) is an early event in breast tumorigenesis. Breast Cancer Res Treat 2004, 88:109-116.

27. Hwang KT, Han W, Cho J, Lee JW, Ko E, Kim EK, Jung SY, Jeong EM, Bae JY, Kang JJ, Yang SJ, Kim SW, Noh DY: Genomic copy number alterations as predictive markers of systemic recurrence in breast cancer. Int J Cancer 2008, 123:1807-1815.

28. Cano CE, Hamidi T, Sandi MJ, Iovanna JL: Nupr1: The swiss-knife of cancer. J Cell Physiol 2011, 226:1439-1443.

29. Ree AH, Tvermyr M, Engebraaten O, Rooman M, Røsok O, Hovig E, Meza-Zepeda LA, Bruland OS, Fodstad O: Expression of a novel factor in human breast cancer cells with metastatic potential. Cancer Res 1999, 59:4675-4680

30. Chowdhury UR, Samant RS, Fodstad O, Shevde LA: Emerging role of nuclear protein 1 (NUPR1) in cancer biology. Cancer Metastasis Rev 2009, 28:225-232.

31. Khoury T, Kanehira K, Wang D, Ademuyiwa F, Mojica W, Cheney R, Morrison C, Conroy J, Nowak N, Liu S: Breast carcinoma with amplified HER2: a gene expression signature specific for trastuzumab resistance and poor prognosis. Mod Pathol 2010, 23:1364-1378.

32. Joensuu H, Isola J, Lundin M, Salminen T, Holli K, Kataja V, Pylkkänen L, Turpeenniemi-Hujanen T, von Smitten K, Lundin J: Amplification of erbB2 and erbB2 expression are superior to estrogen receptor status as risk factors for distant recurrence in PT1N0M0 breast cancer: a nationwide population-based study. Clin Cancer Res 2003, 9:923-930

33. Joerger M, Thürlimann B, Huober J: Small HER2-positive, node-negative breast cancer: who should receive systemic adjuvant treatment? Ann Oncol 2011, 22:17-23.

34. Macrinici V, Romond E: Clinical updates on EGFR/HER targeted agents in early-stage breast cancer. Clin Breast Cancer 2010, 10(Suppl 1):E38-46

35. Yim SH, Kim TM, Hu HJ, Kim JH, Kim BJ, Lee JY, Han BG, Shin SH, Jung SH, Chung YJ: Copy number variations in East-Asian population and their evolutionary and functional implications. Hum Mol Genet 2010, 19:1001-1008.

36. Chin K, DeVries S, Fridlyand J, Spellman PT, Roydasgupta R, Kuo WL, Lapuk A, Neve RM, Qian Z, Ryder T, Chen F, Feiler H, Tokuyasu T, Kingsley C,
Dairkee S, Meng Z, Chew K, Pinkel D, Jain A, Ljung BM, Esserman L, Albertson DG, Waldman FM, Gray JW: Genomic and transcriptional aberrations linked to breast cancer pathophysiologies. Cancer Cell 2006, 10:529-541.

37. Kallioniemi OP, Kallioniemi A, Kurisu W, Thor A, Chen LC, Smith HS, Waldman FM, Pinkel D, Gray JW: ERBB2 amplification in breast cancer analyzed by fluorescence in situ hybridization. Proc Natl Acad Sci USA 1992, 89:5321-5325.

38. Slamon DJ, Clark GM, Wong SG, Levin WJ, Ullrich A, McGuire WL: Human breast cancer: correlation of relapse and survival with amplification of the HER-2/neu oncogene. Science 1987, 235:177-182.

39. Kim YS, Won YS, Park KS, Song BJ, Kim JS, Oh SJ, Jeon HM, Jung SS, Park WC: Prognostic significance of HER2 gene amplification according to stage of breast cancer. J Korean Med Sci 2008, 23:414-420.

doi:10.1186/1471-2407-12-382

Cite this article as: Jung et al:: Simultaneous copy number gains of NUPR1 and ERBB2 predicting poor prognosis in early-stage breast cancer. BMC Cancer 2012 12:382.

\section{Submit your next manuscript to BioMed Central and take full advantage of:}

- Convenient online submission

- Thorough peer review

- No space constraints or color figure charges

- Immediate publication on acceptance

- Inclusion in PubMed, CAS, Scopus and Google Scholar

- Research which is freely available for redistribution 